\title{
Contemporary Perspectives in Nonverbal Behavior: Then and Now
}

\author{
Joann M. Montepare
}

Published online: 7 February 2014

(C) Springer Science+Business Media New York 2014

Since its formal introduction in the research literature in the 1950s and 60s, nonverbal behavior research has witnessed a number of theoretical and methodological developments, described in Patterson's (2014) reflections on historical trends in the field in this special issue on contemporary perspectives in nonverbal behavior. The empirical works also featured in this issue highlight the diverse interests and approaches of current researchers in the field, and reinforce several key qualities (e.g., function, context, pattern, intersection, interaction) in Patterson's view for the need of a more systems-oriented nonverbal framework. More specifically, in their exploration of people's ability to use a deliberate Duchenne smile to persuade another person, Gunnery and Hall (2014) demonstrate how considering the broader functions of nonverbal behaviors can offer instructive opportunities for understanding how particular behaviors operate. Moreover, the importance of the interaction context or setting noted by Patterson is emphasized by their finding that people use a deliberate Duchenne smile only when they are attempting to persuade someone to do something consistent with the actual nature of the persuasion object (e.g., drinking a good tasting beverage) as opposed to persuading someone to do something more contrary (e.g., drinking a bad tasting beverage).

Patterson (2014) further challenges contemporary researchers to focus greater attention on patterns of behavior, as in the real world where interactions are characterized more by the simultaneous initiation of multiple, coordinated behaviors rather than the production of single actions in isolated channels. Farley's (2014) work exploring the role of mimicry, smiling, laughter, body posture, and vocal pleasantness in communicating romantic attraction, relationship-maintenance, and affiliation convincingly supports the value of this invitation. Meadors and Murray's (2014) investigation of implicit racial bias in the reactive nonverbal behaviors of viewers watching videos of Black or White criminal suspects supports Patterson's additional challenge to consider in new ways how nonverbal behavior intersects with culture. As well, the importance of tracking how behavior intersect with

J. M. Montepare ( $₫)$

RoseMary B. Fuss Center for Research on Aging and Intergenerational Studies, Lasell College, 1844 Commonwealth Avenue, Newton, MA 02466, USA

e-mail: jmontepare@lasell.edu 
gender is exemplified in differences observed in the stronger reactions of women versus men, suggesting socialized differences in expressing emotions in certain highly charged contexts. The work of Farley, as well as Meadors and Murray, also calls for contemporary perspectives on nonverbal behavior to compare more systematically the functions and consequences of conscious nonverbal actions alongside those expressed automatically, unintentionally, and non-consciously. Building on Patterson's suggestion, new work would be served well by also considering the mechanisms which guide the simultaneous communication of conscious and unconscious nonverbal actions.

As Patterson (2014) notes, early work in the field focused on single, isolated nonverbal behaviors or channels, reflecting in large part limitations in the technology available to researchers to capture, code, and evaluate behavior. The demonstration offered by Frauendorfer et al. (2014) utilizing state-of-the-art nonverbal social sensing technology to assess nonverbal cues researchers previously coded laboriously by hand shows us not only how far technology has come, but also what fine-grained empirical undertakings are now possible. Certainly, the value of automated approaches must rely on the availability of theoretically convincing algorithms and machine learning technologies. However, the expansion of such approaches offers exciting new opportunities for nonverbal researchers to collaborate with computer scientists and others toward an even broader systems-oriented framework.

Nonverbal research and theory has come a long way since the publication of seminal studies on proxemics, body gestures, and facial expressions five decades ago. The research in this special issue provides a thought-provoking look at contemporary investigations, and exemplifies characteristics which a contemporary understanding of nonverbal behavior challenges us to integrate even further in the years to come.

\section{References}

Farley, S.D. (2014). Nonverbal reactions to an attractive stranger: The role of mimicry in communicating preferred social distance. Journal of Nonverbal Behavior. doi:10.1007/s10919-014-0174-4.

Frauendorfer, D., Schmid Mast, M., Nguyen, L. \& Gatica-Perez, D. (2014). Nonverbal social sensing in action: Unobtrusive recording and extracting of nonverbal behavior in social interactions illustrated with a research example. Journal of Nonverbal Behavior. doi:10.1007/s10919-014-0173-5.

Gunnery, S. D., \& Hall, J. A. (2014). The Duchenne smile and persuasion. Journal of Nonverbal Behavior. doi:10.1007/s10919-014-0177-1.

Meadors, J. D., \& Murray, C. B. (2014). Measuring nonverbal bias through body language responses to stereotypes. Journal of Nonverbal Behavior. doi:10.1007/s10919-013-0172-y.

Patterson, M. L. (2014). Reflections on historical trends and prospects in contemporary nonverbal research. Journal of Nonverbal Behavior. doi:10.1007/s10919-013-0171-z. 